\title{
Herbal Material (Unprocessed) Dosage Form Category
}

National Cancer Institute

\section{Source}

National Cancer Institute. Herbal Material (Unprocessed) Dosage Form Category. NCI

Thesaurus. Code C149558.

A type of solid pharmaceutical dose form consisting of whole, broken or fragmented plants or parts of plants (including algae, fungi, lichen) in an unprocessed state, and which may be dried or fresh. 